\title{
Signal Stability in Periodically Amplified Fiber Transmission Systems Using Multiple Quantum Well Saturable Absorbers for Regeneration
}

\author{
Edman Burr, Member, IEEE, Marianna Pantouvaki, Member, IEEE, Martyn Fice, Member, IEEE, Russell Gwilliam, \\ Andrey Krysa, John Roberts, Associate Member, IEEE, and Alwyn Seeds, Fellow, IEEE
}

\begin{abstract}
The use of multiple quantum well (MQW) saturable absorbers (SAs) for signal regeneration in periodically amplified fiber transmission systems is explored. A systematic study of signal destabilization resulting from incomplete saturation of MQW SAs used for regeneration, and of means of overcoming such destabilization, is presented.

A computer model for MQW SAs, which considers the asymmetric Fabry-Pérot (AFP) cavity structure commonly employed to increase the contrast of such devices, is presented. The model is used to simulate nitrogen-implanted MQW SAs with $<5$ ps recovery time in a transmission system. A comparison is made with results previously obtained for a $10 \mathrm{~Gb} / \mathrm{s}$ standard single-mode fiber (SMF) recirculating loop transmission experiment using MQW SAs and temporary soliton propagation for signal regeneration. The simulations allow the benefits derived from the two parts of the regenerator to be identified, as well as their contributions to the destabilization of the propagating signal. The error-free transmission distance is improved from $\sim 2000$ to $>7000 \mathrm{~km}$ when the two components are combined.
\end{abstract}

Index Terms-Ion implantation, nonlinear optics, optical communication, quantum well devices, saturable absorbers (SAs), 2R regeneration.

\section{INTRODUCTION}

$\mathbf{P}$ ERIODIC optical amplification allows transmission of data over many thousands of kilometers in optical fiber transmission systems. The performance of amplified systems is primarily limited by the accumulation of amplifier noise.

A saturable absorber (SA) is a device exhibiting optical nonlinearity such that its attenuation decreases in response to high optical powers, as shown schematically in Fig. 1. This behavior is typical of saturable absorption due to excitonic absorption bleaching $(\mathrm{EAB})$ in multiple quantum well (MQW) materials. Excitonic absorption can become negligible in the

Manuscript received October 27, 2004; revised September 7, 2005. This work was supported by the Engineering and Physical Sciences Research Council and the European Union.

E. Burr is with Azuro Limited, St. John's Innovation Centre, Cambridge, CB4 0WS, U.K.

M. Pantouvaki, M. Fice, and A. Seeds are with the Department of Electronic and Electrical Engineering, University College London, London WC1E 7JE, U.K. (e-mail: a.seeds@ee.ucl.ac.uk).

R. Gwilliam is with the Surrey Centre for Research in Ion Beam Applications, University of Surrey, Guildford, Surrey GU2 7RX, U.K.

A. Krysa and J. Roberts are with the EPSRC National Centre for III-V Technologies, University of Sheffield, Sheffield S1 3JD, U.K.

Digital Object Identifier 10.1109/JLT.2005.861924

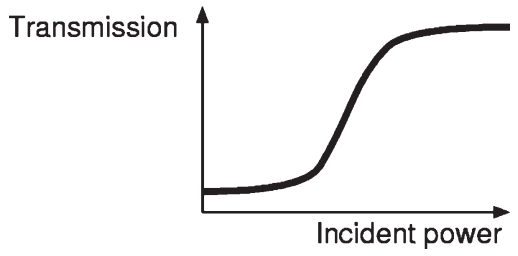

Fig. 1. Schematic SA transfer function.

presence of large carrier populations resulting from intense optical excitation [1].

When incorporated in a return-to-zero (RZ) transmission system, SAs can prevent the accumulation of noise on the zeros, since zeros will experience higher attenuation than the relatively high power signal pulses [2], [3]. Since MQW SAs are passive, they have the potential to provide a simple solution to transmission impairments arising from low-level radiation between pulses.

The use of MQW SAs as regenerators in a $10 \mathrm{~Gb} / \mathrm{s}$ dispersion shifted fiber (DSF) soliton system was proposed in [2] and was extended though numerical simulations to a wavelength division multiplexing (WDM) soliton system, again using DSF, in [4]. The performance improvement was attributed to the prevention of amplified spontaneous emission (ASE) accumulation on the zeros and the attenuation of low-intensity pulse wings (resulting from the soliton-soliton interaction) by the MQW SAs. The regeneration capability of MQW SAs was first demonstrated in a $10 \mathrm{~Gb} / \mathrm{s}$ quasi-linear transmission system in which fiber dispersion was exactly compensated, in [5]. The SAs increased the error-free transmission distance in a $10 \mathrm{~Gb} / \mathrm{s}$ system by a factor of 2.5 when the amplifier noise level was increased.

However, saturation of practical MQW SAs requires the incident optical pulses to have high energy. A saturation energy, defined as the energy resulting in a $50 \%$ absorption reduction in the MQW material, of $12 \mathrm{pJ}$ has been measured in InGaAs/InAlAs and InGaAsP/InGaAsP MQW SAs that have been ion implanted for picosecond recovery [6], [7]. When operating at $10 \mathrm{~Gb} / \mathrm{s}$, or at higher repetition rates, the average output power available from practical erbium-doped fiber amplifiers (EDFAs) will restrict the pulse energies incident on the MQW SAs such that complete saturation is not possible. 
Due to noise and intrachannel or interchannel effects, pulses incident on the SA will have a range of pulse energies. Incomplete MQW SA saturation will result in signal pulses of different energies experiencing differing levels of attenuation, with the attenuation being lower for higher energy pulses. Consequently, the variance in pulse energy will be increased after passing through the SA. A system in which the signal passes through cascaded MQW SAs may therefore exhibit eventual destabilization of the pulse train, that is, dramatically increased noise on ones.

Effective pulse stabilization can be achieved in quasi-linear systems if the signal pulses propagate for some short distance under conditions such that they approximate solitons and are then passed through a narrow bandwidth optical filter [8]. Pulses are transformed into solitons in a DSF. Higher energy solitons experience a greater degree of spectral broadening due to self phase modulation (SPM) as they propagate in the DSF and hence lose more energy in the filter. This technique is referred to as soliton filtering in the rest of this paper. Suitable system parameters will lead to normalization of the energy of the propagating solitons [9]. In its first practical demonstration, soliton filtering was used in conjunction with synchronous amplitude modulation in a two-channel $40 \mathrm{~Gb} / \mathrm{s} / \mathrm{channel}$ WDM system [10]. The soliton filtering technique has not been used in isolation in experimental systems; either MQW SAs [11], [12] or synchronous modulation [13], [14] has been used to prevent the accumulation of ASE on the zeroes.

The use of MQW SAs in conjunction with soliton filtering to improve transmission performance in commercially important standard single-mode fiber (SMF)-based system was first reported in [15]. The configuration, which is discussed in more detail in Section II, used long $(80 \mathrm{~km})$ unamplified spans of SMF between regenerators. Exact dispersion compensation was implemented such that the system operated in the quasi-linear regime. The greater than three times increase of error-free transmission distance observed in the experiments demonstrated that MQW SAs and soliton filtering can improve the performance of nonoptimal systems, which are more representative of those encountered in practical situations. A similar device was used in an earlier demonstration of noise suppression at $40 \mathrm{~Gb} / \mathrm{s}$ [16].

Although techniques have been employed in some of the work reviewed above that have the effect of countering destabilization by the SA, the phenomenon has received little explicit investigation so far. In this paper, we explore in more detail the signal destabilization due to MQW SAs, and the use of soliton filtering to counter it. We present a computer model for MQW SAs based on the asymmetric Fabry-Pérot (AFP) cavity structure and demonstrate its use by simulating previously reported results for a $10 \mathrm{~Gb} / \mathrm{s}$ recirculating loop transmission experiment using MQW SAs and soliton filtering for signal regeneration [12]. This arrangement results in significant increases in error-free transmission distance (defined as the distance to which the signal propagated before the Q-factor fell below 6), from $2000 \mathrm{~km}$ to more than $7000 \mathrm{~km}$ in the $10 \mathrm{~Gb} / \mathrm{s}$ system considered, which employed regeneration after every $80-\mathrm{km}$ span of SMF.

The remainder of the paper is structured as follows. In Section II, the experimental transmission system and the MQW

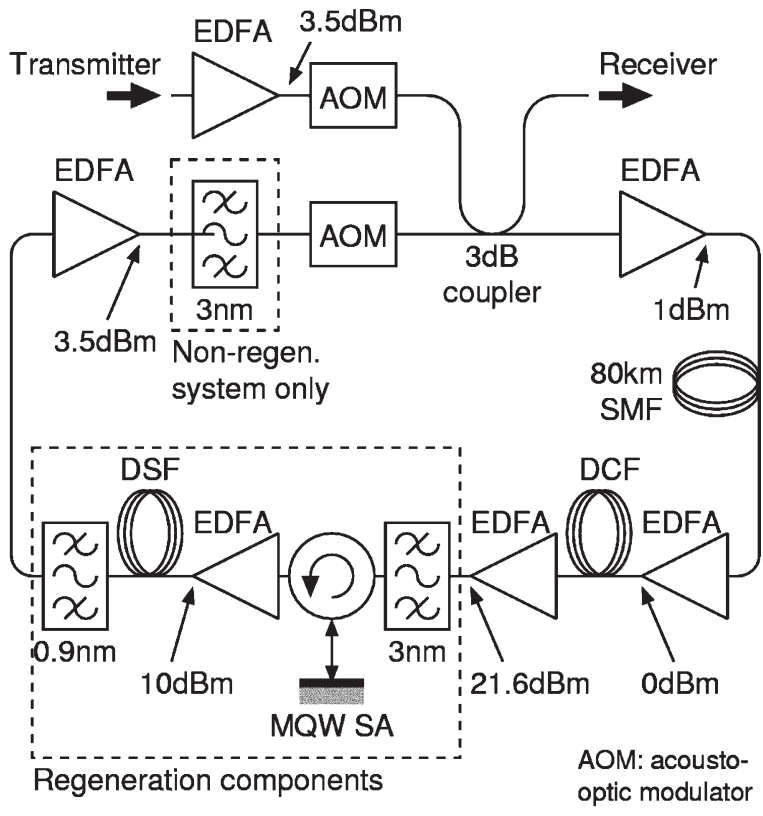

Fig. 2. Ten-gigabit per second recirculating loop configuration.

SA devices used are described. A numerical model of the MQW SAs is developed in Section III, enabling simulation of their use for regeneration in fiber transmission systems. In Section IV, this model is then used to simulate the $10 \mathrm{~Gb} / \mathrm{s}$ transmission system and to investigate the signal destabilization by the SAs and its control by temporary soliton propagation. The main results of the work are discussed in Section V.

\section{EXPERIMENTAL 10-Gb/s SYSTEM}

The systems modeling described in this paper initially focuses on a single-channel $10 \mathrm{~Gb} / \mathrm{s}$ recirculating loop experiment using a reflective MQW SA [12]. The recirculating loop configuration used in the experiments is shown in Fig. 2. The loop contains an $80 \mathrm{~km}$ SMF span with a dispersion of $17 \mathrm{ps} / \mathrm{nm} / \mathrm{km}$. This is compensated for using a length of dispersion compensating fiber (DCF). The dispersion compensator was trimmed to provide exact dispersion compensation such that signal propagation was in the quasi-linear regime. The loop also contains a $3 \mathrm{~km}$ length of DSF through which the signal pulses propagate as solitons, prior to narrowband optical filtering for stabilization. The dispersion of the DSF at the operating wavelength is approximately $4 \mathrm{ps} / \mathrm{nm} / \mathrm{km}$. This soliton filtering mechanism, accomplished by the DSF and the filter, along with the SA, forms the regenerator. The full-width at half-maximum (FWHM) length of the launched pulses is $6 \mathrm{ps}$. When making measurements without the regeneration components, an additional optical filter, with a bandwidth of $3 \mathrm{~nm}$, is inserted in the loop, at the point indicated in Fig. 2. The receiver used in the experiment incorporated a $40 \mathrm{~Gb} / \mathrm{s}$ to $10 \mathrm{~Gb} / \mathrm{s}$ optical demultiplexer.

The structure of the SAs used in the transmission experiment is illustrated schematically in Fig. 3. The MQW region consisted of 50 9.5-nm-thick InGaAsP wells and $517.5-\mathrm{nm}$ thick InGaAsP barriers with band edges at 1.6 and $1.1 \mu \mathrm{m}$, respectively. The Bragg stack had 16 periods, each comprising a quarter wave layer of InP, and a quarter wave layer of InGaAsP 


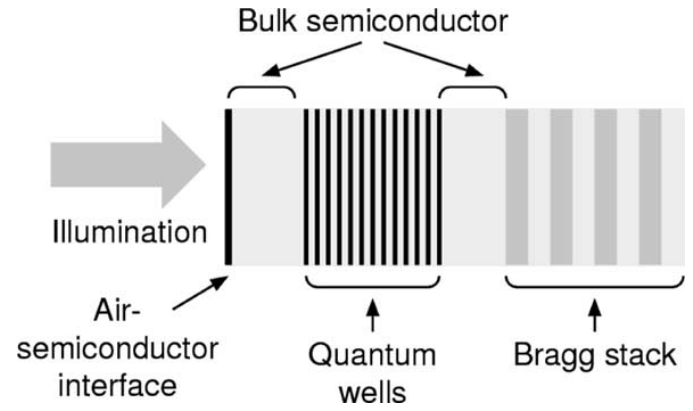

Fig. 3. Schematic of MQW SA device.

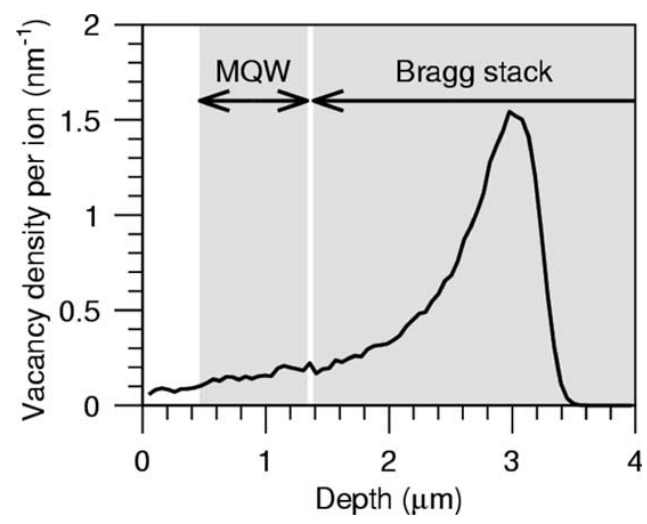

Fig. 4. Simulation results showing the vacancy density per ion resulting from the implantation of the device of Fig. 3 with $4 \mathrm{MeV}$ nitrogen ions.

with a band edge at $1.4 \mu \mathrm{m}$. Both the MQW region and the Bragg stack were grown in a single metal organic vapor phase epitaxy (MOVPE) step [17]. All the materials were lattice matched to the InP substrate to avoid strain.

The Bragg stack and the front air semiconductor interface form an AFP cavity that is resonant at the operating wavelength. Under small signal conditions, the absorption of the MQW region is such that destructive interference between reflections from the AFP cavity results in a small aggregate reflectivity. Optical excitation leads to a reduction of the MQW absorption such that effective destructive interference no longer occurs and the aggregate reflectivity is significantly increased.

Since the absorption changes in the MQW region are a consequence of the EAB optical nonlinearity [1], their speed is dependent on photocarrier lifetime, which is typically several nanoseconds in high quality epitaxially grown MQW structures. Picosecond carrier lifetimes, which are required for $10 \mathrm{~Gb} / \mathrm{s}$ systems, can be achieved in MQW materials containing a suitable population of lattice defects. Mid-gap levels resulting from the presence of defects will accelerate carrier recombination.

To achieve high-speed EAB, nitrogen ions with an energy of $4 \mathrm{MeV}$ were implanted through the top of the device [18]. The vacancy distribution resulting from such ion implantation, as determined using the simulation program SRIM-2000 [19], is shown in Fig. 4. The ions propagate through the MQW region, creating the required defect population, and are deposited deep within the Bragg stack.

Implantation was performed at an elevated temperature of $200{ }^{\circ} \mathrm{C}$. This was sufficient to induce dynamic annealing in

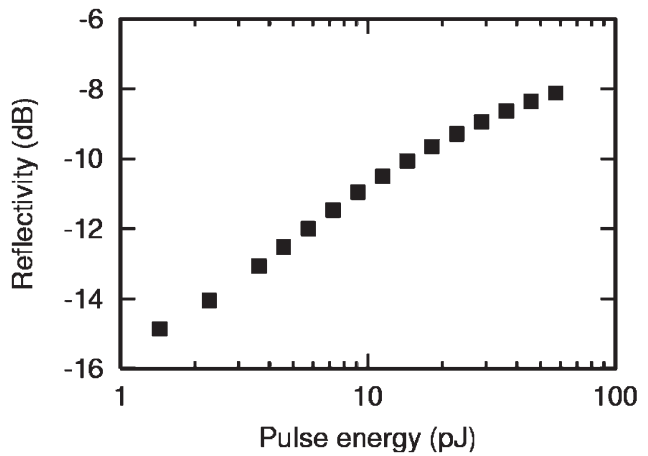

Fig. 5. Dependence of the reflectivity of the MQW SA on pulse energy in measurements conducted using 2 ps duration pulses.

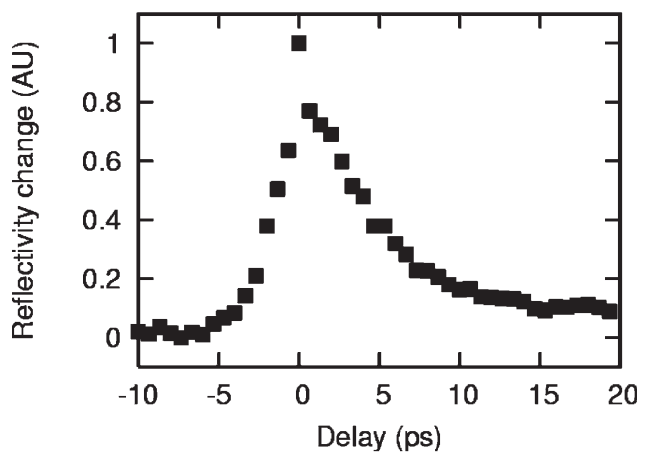

Fig. 6. Results of pump-probe measurement showing the recovery of the ionimplanted MQW SA following saturation by a 2 ps pump pulse.

TABLE I

MQW SA PARAMETERS

\begin{tabular}{|c|c|}
\hline Recovery time & $5 \mathrm{ps}$ \\
\hline MQW small signal absorption & $5 \times 10^{5} \mathrm{~m}^{-1}$ \\
\hline MQW saturation power & $2 \mathrm{~W}$ \\
\hline
\end{tabular}

the device, limiting damage accumulation and hence preserving the nonlinear response of the MQW [17]. The ion dose used was $3 \times 10^{12} \mathrm{~cm}^{-2}$. This implantation strategy enabled a high-speed high-contrast device to be realized using a standard MOVPE process and with no requirement for postgrowth fabrication.

The nonlinear response of the device is shown in Fig. 5. This plot shows the dependence of reflectivity of the device on pulse energy, measured using 2 ps duration pulses. The recovery dynamics of the nonlinearity in MQW SA were investigated using pump-probe measurements, results from which are shown in Fig. 6. The recovery time of the ion-implanted device, as determined by fitting an exponential curve to the measured data, was less than 4.7 ps. Prior to ion implantation, the recovery time of the device was 3 ns.

\section{SA MODEL}

A model was designed to represent the reflective AFP cavity MQW SA devices used in the transmission system described in the previous section. It calculates the change in reflectivity that results from the incidence of signal pulses. The calculated 


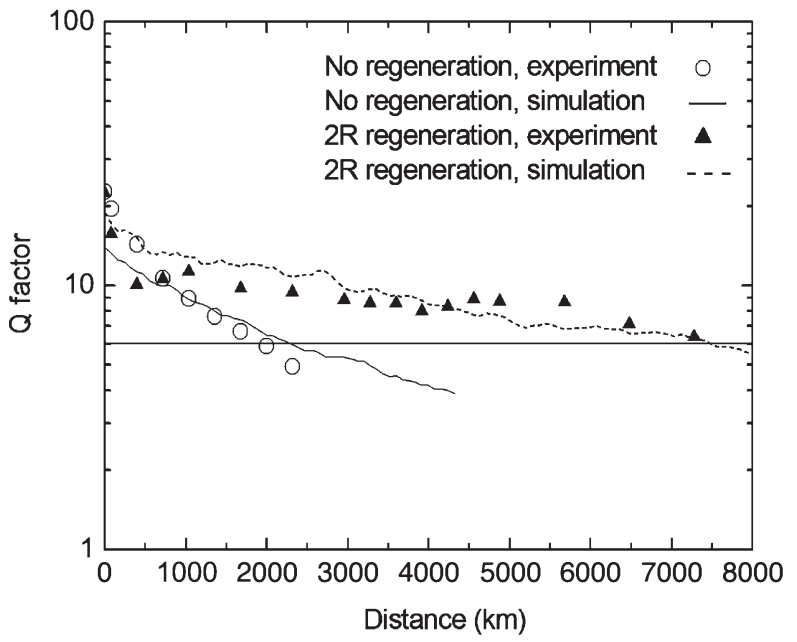

Fig. 7. Q-factor evolution in the $10 \mathrm{~Gb} / \mathrm{s}$ system with no regeneration and using MQW SAs and soliton filtering for regeneration.

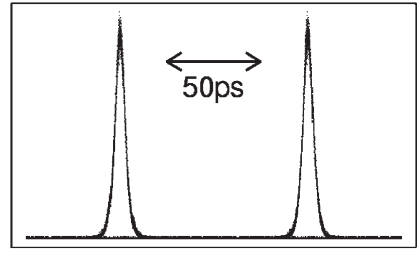

$80 \mathrm{~km}$

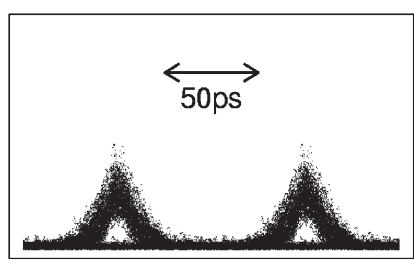

$2000 \mathrm{~km}$
Fig. 8. Eye diagrams illustrating the optical signal in the simulated $10 \mathrm{~Gb} / \mathrm{s}$ system with no regeneration.

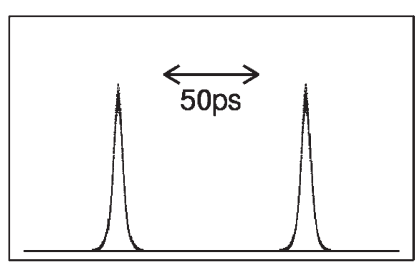

$80 \mathrm{~km}$

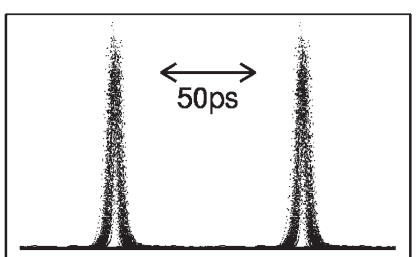

$2000 \mathrm{~km}$
Fig. 9. Eye diagrams illustrating the optical signal in the simulated $10 \mathrm{~Gb} / \mathrm{s}$ system using MQW SAs and soliton filtering for regeneration.

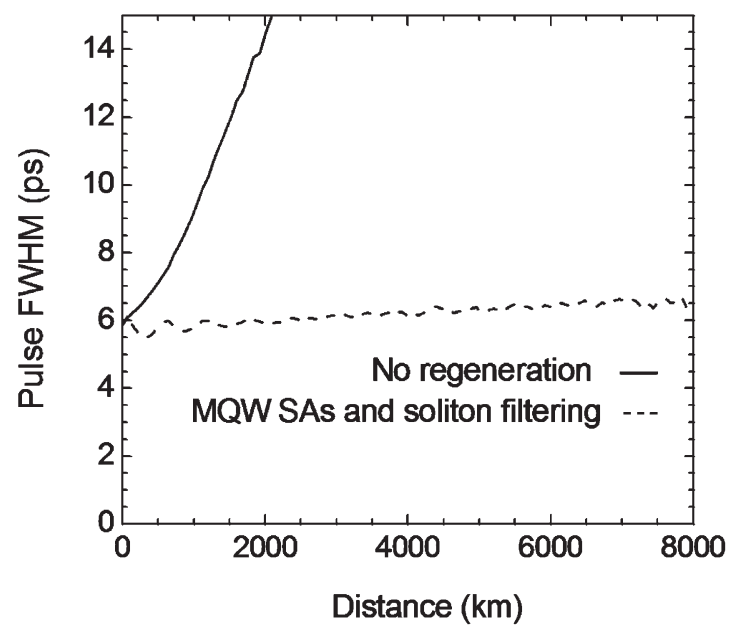

Fig. 10. Evolution of mean pulse duration in the $10 \mathrm{~Gb} / \mathrm{s}$ system using MQW SAs and soliton filtering.

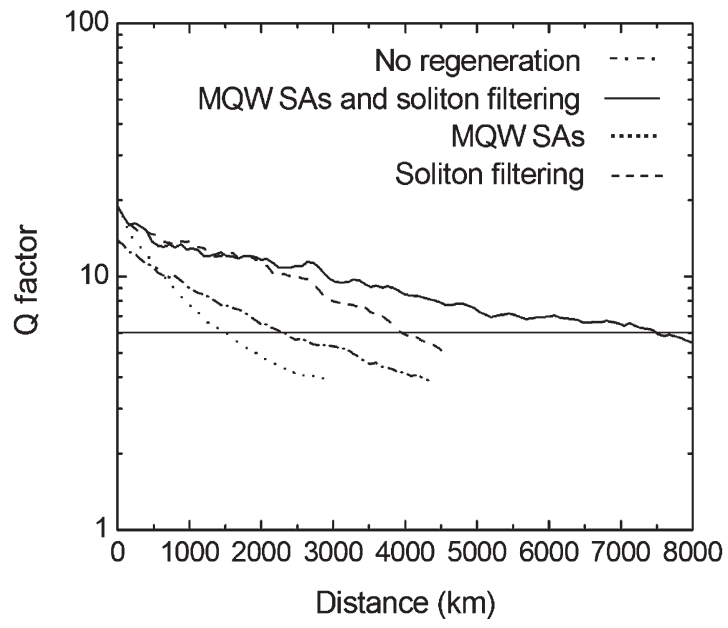

Fig. 11. Q-factor evolution in $10 \mathrm{~Gb} / \mathrm{s}$ simulations for different combinations of MQW SAs and soliton filtering.

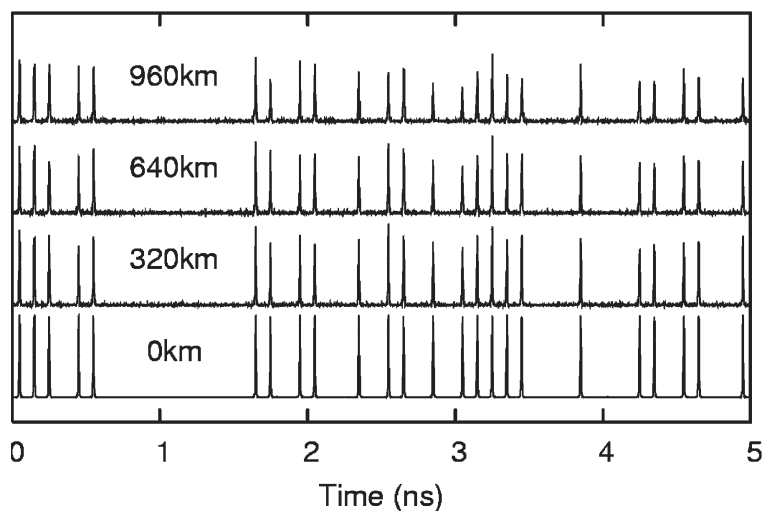

Fig. 12. Evolution of a part of the pulse train in the simulation of the system with MQW SAs only.
SA only

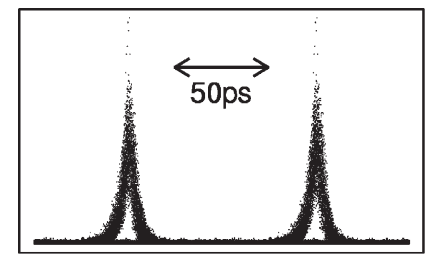

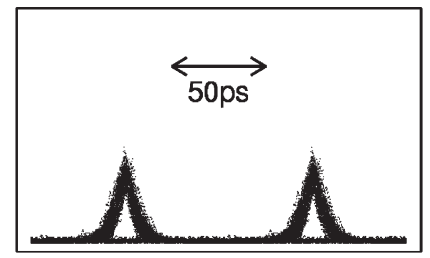

No regeneration.
Fig. 13. Eye diagrams comparing the optical signal after $1200 \mathrm{~km}$ in systems with no regeneration components and with only MQW SAs.

reflectivity can then be used to modify the signal pulses propagating in the modeled transmission system.

MQW SA dynamics can be modeled using a set of rate equations considering populations of photo-generated excitons and free carriers [20]. This approach uses the concept of screening area to represent the reduction of absorption in the MQW due to the presence of the exciton and free photocarrier populations.

Due to the relatively short lifetimes of excitons, screening due to the exciton population need only be considered in the case of sub-picosecond optical excitations [1]. The transmission system modeling described in this paper therefore uses a simplified rate equation model that ignores exciton screening.

The photocarrier population in each of the quantum wells (QWs) within the device is modeled separately. This allows 

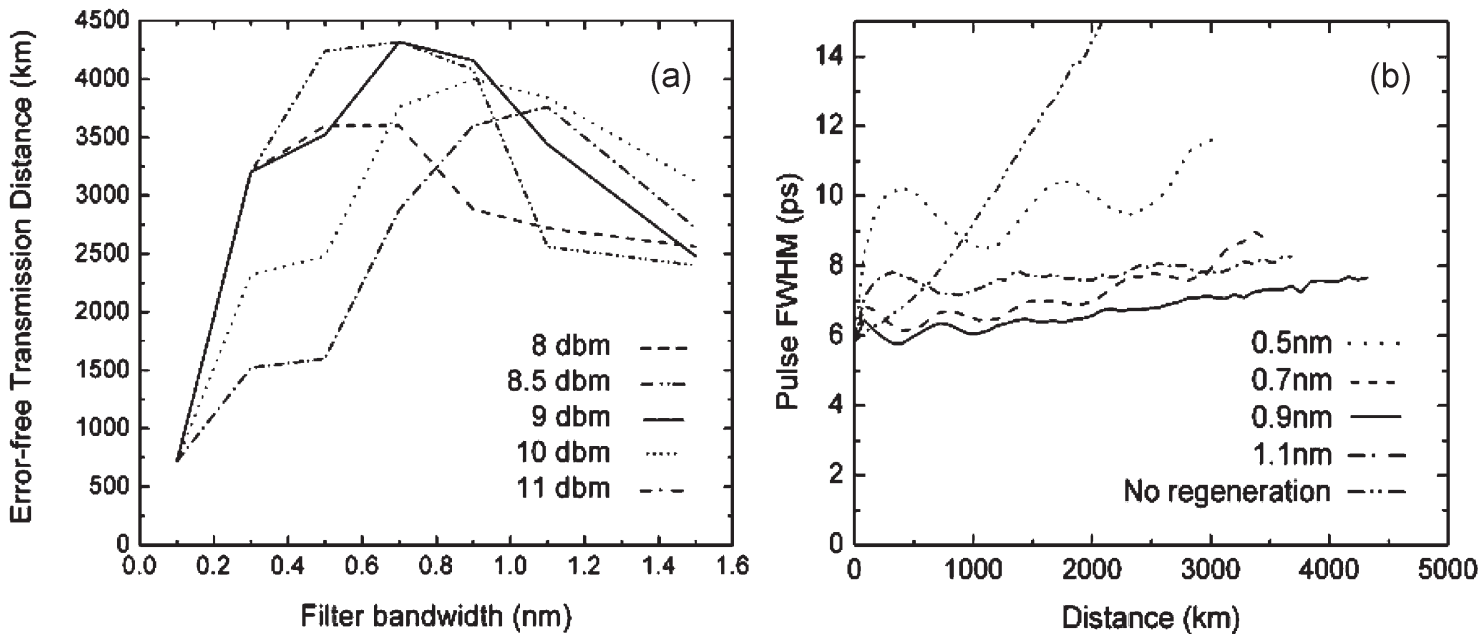

Fig. 14. (a) Error-free transmission distance versus filter bandwidth for different values of the power input to the DSF fiber. (b) Evolution of mean pulse duration in the $10 \mathrm{~Gb} / \mathrm{s}$ system using soliton filtering only, for different filter bandwidths.

the nonuniform power due to the standing wave in the AFP cavity to be accounted for. Populations are modeled using the rate equation

$$
\frac{d x}{d t}=\frac{(1-x)}{\tau P_{S}} P-\frac{x}{\tau}
$$

where $x$ is the normalized photocarrier population, $P$ is the incident optical power, $P_{S}$ is the saturation power, and $\tau$ is the time constant for the exponential recovery time of the photocarrier population. The normalization of $x$ is such that $x=1$ when the QW is fully saturated. $P_{S}$ is defined as the power of unmodulated light that will result in a factor-of-two reduction of the QW absorption. Equation (1) is written in terms of power and saturation power, rather than intensity and saturation intensity as in the case of [20] for compatibility with the transmission system model. Given the value of $x$ in a well, the absorption in that well is $\alpha=\alpha_{0}(1-x)$, where $\alpha_{0}$ is the small signal absorption. The decay term $x / \tau$ assumes exponential decay of the photocarrier population.

Having calculated the well absorptions, the magnitude and phase of the reflectivity of the AFP cavity are determined using scattering matrices [21]. The field due to the standing wave, and hence its power, can be calculated in each well by summing field contributions due to the incident wave as it is reflected back and forth within the cavity.

For systems modeling, the response of the SA to a signal, represented as a series of points in the time domain, must be determined. To achieve this, the following steps are performed for each point in the representation of the incident signal: the absorption in each QW is determined from the corresponding value of the photocarrier population $x$; the power of the standing wave in each well is calculated; the value of $x$ in each well is updated using the corresponding value of $d x / d t$ from (1); and the overall reflectivity of the SA is calculated.

This MQW-AFP SA model was incorporated into a computer program that was used to simulate periodically amplified transmission systems. The split-step Fourier method [22] was used to describe signal propagation in optical fiber. A pseudo- random bit sequence of 256 bits was used. The MQW SA parameters used in the modeling are summarized in Table I. These were set such that the results of the device model were consistent with the characterization results shown in Section III.

\section{Simulation of the Experimental $10 \mathrm{~Gb} / \mathrm{s}$ SYSTEM}

Initial simulations of the $10 \mathrm{~Gb} / \mathrm{s}$ system were done to verify that the results were consistent with those obtained experimentally. Fig. 7 compares simulated and experimentally measured Q-factor evolution without regeneration and with regeneration. There is a satisfactory agreement between results obtained from experiment and from modeling.

Eye diagrams of the optical signal in the simulation without regeneration after propagating once, and 25 times, around the recirculating loop are shown in Fig. 8. After $2000 \mathrm{~km}$, the pulses are broadened, while noise is added on zeros and ones. Pulse broadening is not evident in the corresponding eye diagrams for the simulation with regeneration, shown in Fig. 9. The noise on zeros appears to have been reduced with regeneration, while it is not possible to conclude a trend on the noise on ones.

The pulse width evolution in the system with and without regeneration is shown in Fig. 10. The pulse width in the unregenerated system increases steadily from $6 \mathrm{ps}$ at launch to more than $14 \mathrm{ps}$ after propagating for $2000 \mathrm{~km}$. The pulse width in the regenerated system is generally preserved, increasing only to about $6.5 \mathrm{ps}$ as it reaches $7000 \mathrm{~km}$. The relatively stable pulse width in the regenerated system is dependent on the parameters for temporary soliton propagation in DSF, the launch power, group velocity dispersion (GVD), and the bandwidth of the filter following the DSF.

To investigate the effect of MQW SAs and soliton filtering in the system individually, the Q-factor evolution is plotted in Fig. 11. In the simulations using only SAs, there is no filter incorporated after the MQW SA. While some improvement of performance is seen in the system using only soliton filtering, there is a dramatic degradation in the system using only MQW 
SAs. However, when the two components are combined, the error-free transmission distance is increased by a factor of 1.9 compared to soliton filtering alone.

Fig. 12 shows the evolution of a part of the pulse train in the simulation with MQW SAs only in the system. A significantly greater variation of the levels of ones is observed as the signal passes multiple times through the loop. Small energy variations acquired by the propagating signal pulses, due to noise, or to intrachannel cross phase modulation [23], are amplified after transmission through the MQW SA. The eye diagrams of Fig. 13 compare the signals in the simulation using MQW SAs for regeneration, and in that with no regeneration, after 15 trips round the loop. It is shown that the variation of the levels of the ones, which occurs when MQW SAs are used, is not as significant in the eye diagrams without regeneration.

Fig. 14(a) shows the error-free transmission distance achieved in the system with soliton filtering only. For these simulations the DSF parameters were kept the same, while the filter bandwidth and the power into the fiber were varied. It was found that the spectral filtering used in the $3 \mathrm{~km}$ span of DSF was not far from optimum in the $10 \mathrm{~Gb} / \mathrm{s}$ experiment of [12], where the power into the fiber was $10.5 \mathrm{dBm}$. The pulse-width evolution for a few filter bandwidths at this power is plotted in Fig. 14(b). Temporary soliton propagation in the $3 \mathrm{~km}$ length of DSF and optical filtering with a bandwidth of $0.9 \mathrm{~nm}$ controls the pulse width and limits pulse broadening significantly in this dispersion compensated system.

A better understanding of the system using soliton filtering only, and both soliton filtering and MQW SAs can be gained by referring to Fig. 15, which shows a representation of the evolution of a short section of the signal as it propagates 50 times around the loop. Results without regeneration, and using MQW SAs as well as soliton filtering, are shown for comparison. Preservation of the input pulse width occurs in the result with soliton filtering; however, there is also noise accumulation in the intervals between the signal pulses, which gradually degrades the system performance. MQW SAs remove this noise, resulting in stable pulse transmission in the case of full regeneration.

In order to isolate the effect of each component on zeros and ones, the partial Q-factors $Q_{1}$ on ones and $Q_{0}$ on zeros are plotted on Fig. 16. $Q_{1}$ and $Q_{0}$ are calculated after each transmission through the loop as described in [24], that is

$$
Q_{1}=\frac{\mu_{1}-D}{\sigma_{1}} \text { and } Q_{0}=\frac{D-\mu_{0}}{\sigma_{0}}
$$

where $\mu_{1,0}$ and $\sigma_{1,0}$ are the mean and the standard deviation of ones and zeros, respectively, and $D$ is the decision voltage.

Fig. 16 shows that when used alone, MQW SAs add noise on ones while they suppress the noise on zeros. The destabilization of the ones occurs very rapidly, with significant impact after only a few passes through the SA (Fig. 12), while the noise on the zeros only becomes significant after a much larger number of recirculations around the loop, so there is a reduction of the overall Q-factor compared to the case without regeneration (Fig. 11). However, when combined with some mechanism of pulse stabilization, such as soliton filtering, the adverse effect of
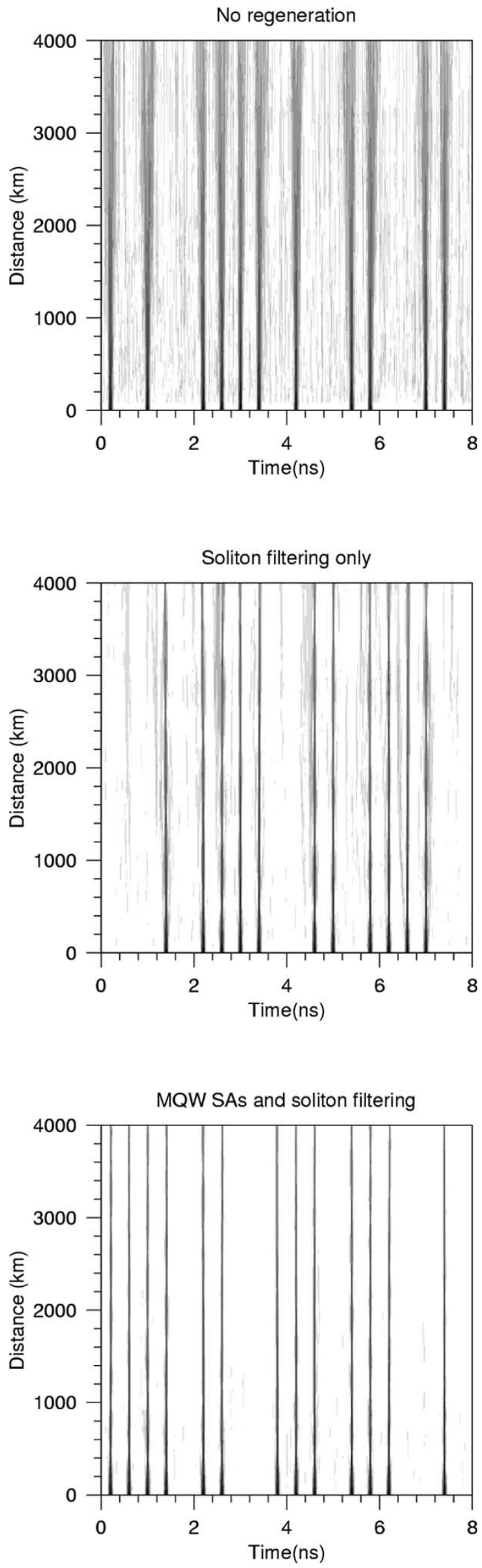

Fig. 15. Evolution of representative section of the propagating pulse streams. 

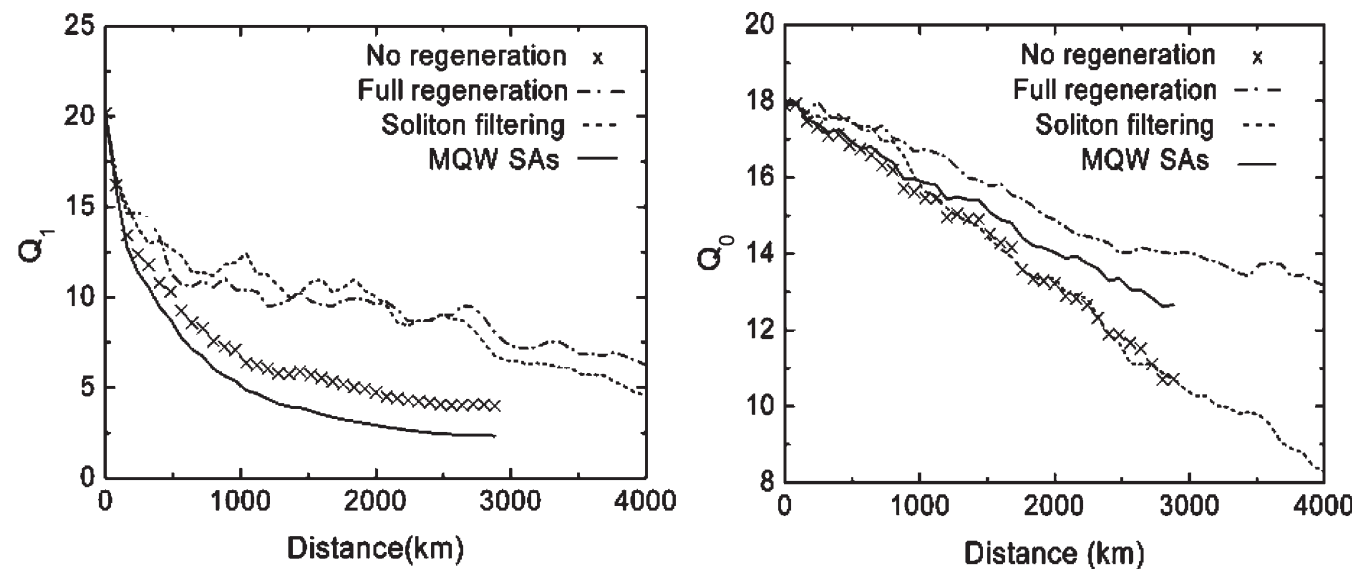

Fig. 16. $Q_{1}$ and $Q_{0}$ plotted versus transmission distance.

MQW SAs is cancelled and the overall transmission is further improved due to noise suppression on ones by the SAs.

The results presented in this section are consistent with the assertion that, in a system in which MQW SAs are to be used to prevent noise accumulation on the zeros, some mechanism, such as soliton filtering, is required to stabilize the signal pulses.

\section{CONCLUSION}

A detailed MQW SA model for AFP cavity devices has been developed, allowing the power of the standing wave in the AFP cavity and the dynamics of recovery from saturation in the MQW to be included in simulations of transmission systems. This model was used in a number of simulation exercises. These focused on the recirculating loop configuration used in a $10 \mathrm{~Gb} / \mathrm{s}$ transmission experiment. In this experiment, MQW SAs and soliton filtering were shown to significantly extend the error-free distance in a quasi-linear system with $80 \mathrm{~km} \mathrm{SMF}$ spans between regenerators.

The model was used to investigate the relative contributions of the different elements of the regeneration process. The behavior of the system was studied when regeneration was performed using MQW SAs or soliton filtering separately, and when they were combined.

When used in isolation, MQW SAs prevented noise accumulation in the intervals between signal pulses. However, the rapid destabilization of signal pulses in the system studied led to an overall degradation of the system performance. The errorfree transmission distance (defined as the distance to which the signal propagated in the simulated system before the Q-factor fell below 6) was reduced from $2240 \mathrm{~km}$ in the absence of regeneration to $1440 \mathrm{~km}$ with MQW SAs only, due to noise accumulation on the ones.

With soliton filtering alone, the error-free transmission distance was $3920 \mathrm{~km}$ : an increase by a factor of 1.75 over the distance without regeneration. This was due to a reduction in the noise on the ones. Soliton filtering was also shown to stabilize the pulse width.

When MQW SAs and soliton filtering were combined, noise levels on both ones and zeros were significantly reduced compared to those without regeneration, resulting in an error-free transmission distance of $\sim 7500 \mathrm{~km}$ : an increase by a factor of 3.3 over the distance without regeneration. Thus, when used in concert with a technique that stabilizes the pulse amplitude (ones), MQW SAs are shown to be a highly effective method for regeneration.

The soliton filtering technique investigated here for pulse stabilization requires an additional amplification stage, and the length of DSF required significantly increases the physical size of the regenerator. Future research could thus be usefully directed toward the development of alternative compact devices with limiting behavior suitable for pulse stabilization, and SAs with reduced saturation power.

\section{REFERENCES}

[1] D. S. Chemla and D. A. B. Miller, "Room-temperature excitonic nonlinear-optical effects in semiconductor quantum-well structures," J. Opt. Soc. Amer. B, Opt. Phys., vol. 2, no. 7, pp. 1155-1173, Jul. 1985.

[2] D. Atkinson, W. H. Loh, V. V. Afanasjev, A. B. Grudinin, A. J. Seeds, and D. N. Payne, "Increased amplifier spacing in a soliton system with quantum-well saturable absorbers and spectral filtering," Opt. Lett., vol. 19, no. 19 , pp. 1514-1516, Oct. 1994.

[3] M. Matsumoto, H. Ikeda, T. Uda, and A. Hasegawa, "Stable soliton transmission in the system with nonlinear gain," J. Lightw. Technol., vol. 13, no. 4, pp. 658-665, Apr. 1995.

[4] O. Audouin, E. Pallise, E. Desurvire, and E. Maunand, "Use of fast in-line saturable absorbers in wavelength division multiplexed soliton systems," IEEE Photon. Technol. Lett., vol. 10, no. 6, pp. 828-829, Jun. 1998.

[5] J. Mangeney, S. Barre, G. Aubin, J. L. Oudar, and O. Leclerc, "System application of $1.5 \mu \mathrm{m}$ ultrafast saturable absorber in $10 \mathrm{~Gb} / \mathrm{s}$ long-haul transmission," Electron. Lett., vol. 36, no. 20, pp. 1725-1727, Sep. 2000.

[6] J. Mangeney, J. L. Oudar, J. C. Harmand, C. Meriadoc, G. Patriarche, and G. Aubin, "Ultrafast saturable absorption at $1.55 \mu \mathrm{m}$ in heavy-ionirradiated quantum-well vertical cavity," Appl. Phys. Lett., vol. 76, no. 11, pp. 1371-1373, Mar. 2000.

[7] E. P. Burr, M. Pantouvaki, A. J. Seeds, R. J. Gwilliam, S. M. Pinches, and C. C. Button, "Wavelength conversion of $1.53 \mu \mathrm{m}$ wavelength picosecond pulses in an ion-implanted multiple-quantum-well all-optical switch," Opt. Lett., vol. 28, no. 6, pp. 483-485, Mar. 2003.

[8] P. Brindel, B. Dany, O. Leclerc, and E. Desurvire, "Black box optical regenerator for RZ transmission systems," Electron. Lett., vol. 35, no. 6, pp. 480-481, Mar. 1999.

[9] M. Matsumoto, "Time-domain transmission control of dispersionmanaged solitons," Electron. Lett., vol. 34, no. 22, pp. 2155-2157, Oct. 1998

[10] O. Leclerc, P. Brindel, D. Rouvillain, B. Dany, E. Pincemin, E. Desurvire, C. Duchet, A. Shen, F. Blache, A. Coqielin, M. Goix, S. Bouchoule, and P. Nouchi, " $2 \times 40$ Gbit/s WDM optically regenerated dispersion-managed transmission over $10000 \mathrm{~km}$ with narrow channel spacing," Electron. Lett., vol. 36, no. 1, pp. 58-59, Jan. 2000. 
[11] D. Rouvillain, F. Seguinneau, L. Pierre, P. Brindel, H. Choumane, G. Aubin, J. L. Oudar, and O. Leclerc, " $40 \mathrm{Gbit} / \mathrm{s}$ optical 2R regenerator based on passive saturable absorber for WDM long-haul transmission," in Proc. Optical Fiber Communications Conf. Postconf. Tech. Dig., Anaheim, CA, Apr. 2002, p. FD11-1. Postdeadline Papers.

[12] M. Pantouvaki, M. J. Fice, R. Feced, E. P. Burr, R. Gwilliam, A. B. Krysa, J. S. Roberts, and A. J. Seeds, " $10 \mathrm{~Gb} / \mathrm{s}$ all-optical $2 \mathrm{R}$ regeneration using an MQW Fabry-Pérot saturable absorber and a nonlinear fiber," IEEE Photon. Technol. Lett., vol. 16, no. 2, pp. 617-619, Feb. 2004.

[13] B. Dany, P. Brindel, O. Leclerc, and E. Desurvire, "Transoceanic $4 \times 40 \mathrm{Gbit} / \mathrm{s}$ system combining dispersion-managed soliton transmission and new 'black-box' in-line optical regeneration," Electron. Lett., vol. 35, no. 5, pp. 418-420, Mar. 1999.

[14] A. Sahara, T. Inui, T. Komukai, H. Kubota, and M. Nakazawa, " 40 Gb/s $\mathrm{RZ}$ transmission over transoceanic distance in a dispersion managed standard fiber using a new inline synchronous modulation method," IEEE Photon. Technol. Lett., vol. 12, no. 6, pp. 720-722, Jun. 2000.

[15] M. Pantouvaki, E. P. Burr, A. J. Seeds, and J. S. Roberts, "Enhanced $10 \mathrm{~Gb} / \mathrm{s}$ error-free transmission distance using all optical $2 \mathrm{R}$ regeneration with ion-implanted MQW saturable absorber and non-linear fibre," in Proc. Conf. Lasers and Electro-Optics, Baltimore, MD, May 2003, pp. 1927-1928.

[16] M. Pantouvaki, E. Burr, R. Feced, M. Fice, R. Gwilliam, J. S. Roberts, and A. J. Seeds, " $40 \mathrm{~Gb} / \mathrm{s}$ optical noise suppression and wavelength conversion by MQW saturable absorber integrated in a Fabry-Pérot cavity," presented at the Eur. Conf. Optical Communications, Copenhagen, Denmark, Sep. 2002, Paper 6.3.6.

[17] M. Pantouvaki, R. Gwilliam, E. P. Burr, A. B. Krysa, J. S. Roberts, and A. J. Seeds, "Ultrafast recovery times and increased absorption nonlinearity in InGaAsP MQW saturable absorbers implanted at $200{ }^{\circ} \mathrm{C}$," in Proc. Int. Conf. Indium Phosphide and Related Materials, Santa Barbara, CA, May 2003, pp. 355-358.

[18] E. L. Delpon, J. L. Oudar, N. Bouche, R. Raj, A. Shen, N. Stelmakh, and J. M. Lourtioz, "Ultrafast excitonic saturable absorption in ion-implanted InGaAs/InAlAs multiple quantum wells," Appl. Phys. Lett., vol. 72, no. 7, pp. 759-761, Feb. 1998.

[19] J. F. Ziegler, J. P. Biersack, and U. Littmark, The Stopping and Range of Ions in Solids. New York: Pergamon, 1985.

[20] H. A. Haus and Y. Silberberg, "Theory of mode locking a laser diode with a multiple quantum well structure," J. Opt. Soc. Amer. B, Opt. Phys., vol. 2, no. 7, pp. 1237-1243, Jul. 1985.

[21] A. E. Siegman, Lasers. New York: University Science, 1986.

[22] G. P. Agrawal, Nonlinear Fibre Optics, 2nd ed. San Diego, CA: Academic, 1995

[23] R.-J. Essiambre, B. Mikkelsen, and G. Raybon, "Intra-channel crossphase modulation and four-wave mixing in high-speed TDM systems," Electron. Lett., vol. 35, no. 18, pp. 1576-1578, Sep. 1999.

[24] N. S. Bergano, F. W. Kerfoot, and C. R. Davidson, "Margin measurements in optical amplifier systems," IEEE Photon. Technol. Lett., vol. 5, no. 3, pp. 304-306, Mar. 1993.

Edman Burr (S'00-A'01-M'04) was born in Bishop's Stortford, U.K., in 1974. He received the B.Sc. degree in physical electronics from the University of Warwick, Coventry, U.K., in 1995 and the Ph.D. degree in 2004.

He spent two years as a Design Engineer with Racal Research Ltd., working in the application-specified integrated circuit (ASIC) design and data-communications systems groups, prior to seven years with the UltraFast Photonics Group at University College London, London, U.K. His interests in photonics include modeling of optical fiber transmission systems, nonlinear optical effects in multiple quantum well devices, and the use of such devices for systems applications including signal regeneration and wavelength conversion. He is now with Azuro, Cambridge, U.K.

Dr. Burr is a member of the Institution of Electrical Engineers.

Marianna Pantouvaki (M'05) received the degree in physics from the University of Athens, Athens, Greece, in 1998 and the M.Sc. degree in telecommunications and the Ph.D. degree in electronic engineering from University College London, London, U.K., in 1999 and 2004, respectively. Her Ph.D. research concerned multiple quantum well saturable absorbers with picosecond recovery times for high bit rate optical communications.

She currently holds the position of Research Fellow at the Ultra-Fast Photonics Group, University College London, working on rapidly tunable semiconductor lasers, optical comb generators, and implanted integrated saturable absorbers for all-optical processing.
Martyn Fice (S'86-M'87) received the B.A. degree in electrical sciences and the Ph.D. degree in microelectronics from the University of Cambridge, Cambridge, U.K., in 1984 and 1989, respectively.

In 1989, he joined STC Technology Laboratories, Harlow, U.K. (later acquired by Nortel Corporation, Harlow), working for several years on the design and development of InP-based semiconductor lasers for undersea optical systems and other applications. Subsequent work at Nortel Corporation involved research into various aspects of optical communications systems and networks, including wavelength division multiplexing (WDM) transmission, all-optical wavelength conversion, optical regeneration, and optical packet switching. $\mathrm{He}$ joined the Photonics Group at University College London, London, U.K., in 2005 and is currently working on optical phase-locked loop techniques.

Dr. Fice is a member of the Institution of Electrical Engineers.

Russell Gwilliam, photograph and biography not available at the time of publication.

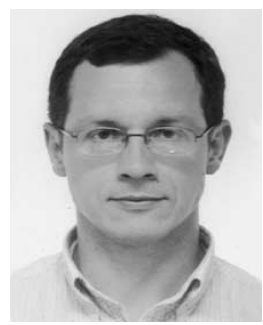

Andrey Krysa graduated from the Moscow Engineering Physics Institute, Moscow, Russia, in 1990. $\mathrm{He}$ received the Ph.D degree in solid state physics from the Lebedev Physical Institute of Russian Academy of Sciences, Moscow, in 1997.

In 1995, he joined the Department of Optoelectronics at the Lebedev Physical Institute as a Research Associate. From 1999 to 2000, he was with the Institute für Halbleitertechnik, RWTH Aachen, Aachen, Germany, as a Humboldt Research Fellow, working on the problem of $\mathrm{ZnSe}$ homoepitaxial growth by MOVPE. Since joining the EPSRC National Centre for III-V Technologies at the University of Sheffield, Sheffield, U.K., in 2001, he has been engaged in the MOVPE of the group III phosphides and arsenides.

John Roberts (M'03-A'03) received the degree in applied chemistry and the $\mathrm{Ph} . \mathrm{D}$. degree in radiation chemistry in 1971 and 1975, respectively.

From 1975 to 1980, he was employed by Philips Research Laboratories, Redhill, U.K., developing the molecular beam epitaxial growth of GaInP for laser applications. From 1980 to the present, he has occupied positions within the EPSRC National Centre for III-V Technologies and is currently responsible for the metal-organic vapor phase epitaxial growth of arsenide and phosphide materials.

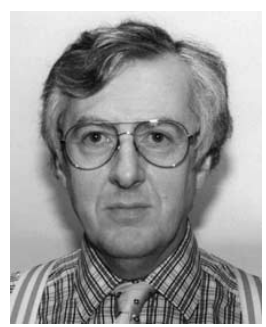

Alwyn Seeds (M'81-SM'92-F'97) received the Ph.D. and D.Sc. degrees from the University of London, London, U.K.

From 1980 to 1983 , he was a Staff Member at Lincoln Laboratory, Massachusetts Institute of Technology, Cambridge, where he worked on GaAs monolithic millimeter-wave integrated circuits. $\mathrm{He}$ returned to England in 1983 to take up a lectureship in telecommunications at Queen Mary College, University of London, moving to University College London in 1986, where he is now Professor of Optoelectronics and Head of the Photonics Group. He has published over 200 papers on microwave and opto-electronic devices and their systems applications. His current research interests include ultra-fast tuneable semiconductor lasers, optical regenerators, semiconductor optical modulators, optical frequency synthesis, broadband radio over fiber access systems, and terahertz photonic systems.

Prof. Seeds is Chairman of the IEE Photonics Professional Network, a Fellow of the Royal Academy of Engineering, a member of the Microwave Photonics Committee of IEEE LEOS, and a member of the Steering Committee for the IEEE International Topical Meeting on Microwave Photonics. He has edited a number of journal special issues on microwave photonics and has served on the programme committees for many international conferences. 